\title{
Monolithic Digital Magnetic Hall Sensor with two Measurement Paths for an ASIL D Application
}

\author{
Friedrich Rasbornig ${ }^{1}$, Wolfgang Granig ${ }^{1}$, Joao Cunha ${ }^{2}$ \\ ${ }^{1}$ Infineon Technologies Austria AG, Siemensstraße 2, 9500 Villach, Austria \\ ${ }^{2}$ Infineon Technologies AG, Am Campeon 1-12, 85579 Neubiberg, Germany \\ friedrich.rasbornig@infineon.com
}

\begin{abstract}
Since the ISO26262 standard [1] was introduced in 2011 in the automotive industry the requirements regarding functional safety are significantly increased, especially for semiconductor products. An increased amount of semiconductor devices are used in safety-critical applications even with the highest ASIL rating (ASIL D) by usage of homogenous redundancy of two separated products or two silicon dies within one chip-package [2] [3].

In this paper a new product concept will be presented for an integrated linear Hall sensor fulfilling the highest ASIL rating. The basic concept is to implement two redundant and diverse measurement paths on one monolithic silicon die to achieve the functional safety requirements and the safety concept for ASIL D requirements. Also the evaluation results based on laboratory measurements of the sensor product on silicon level will be shown.
\end{abstract}

An overview of the main automotive applications will be presented, where this product will be used. The main application for this product is the electronic power steering (EPS) application, where the torque is measured via the magnetic field size and via a certain magnetic setup transferring this torque into a magnetic field size and further converted to the corresponding digital output values of the linear Hall sensor.

Key words: Functional Safety, ASIL D, ISO26262, Electric Power Steering, Hall Sensor

\section{Overview of Electronic Power Steering Application (ASIL D)}

Electric power steering uses an electric motor to assist the steering of a vehicle. A sensor detects the torque exerted on the steering wheel by the driver and an electronic control unit (ECU) applies assisted torque via the motor, which connects to either steering gear or steering column.

The following safety goals apply for the EPS system:

- Avoid self-steering, ASIL D

- Avoid blocked steering, ASIL D

- Avoid sudden loss of steering assist, ASIL B

\section{Fault-Tolerant Electric Power Steering systems}

When EPS was introduced, the mechanical connection between steering wheel and steering bar was seen as a valid backup option for manual steering and thus a shutdown of the steering assistance was considered a safe state of the system.

This judgement is no longer accepted by most OEMs, considering that the average driver will most likely not be able to control a car in case the steering assistance suddenly fails.

Therefore, the safety goal "avoid sudden loss of steering assistance, ASIL B" was introduced, this requires from a safety point of view, only the implementation of additional mechanisms that provide sufficient steering assistance functionality for a very limited amount of time. 


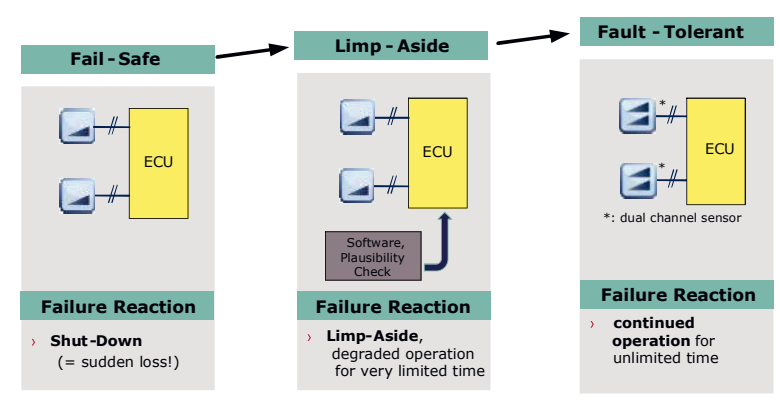

Fig. 1. Transition from Fail-Safe to Fault-Tolerant EPS

However, as such failures of "limp-aside" systems pose a major discomfort for the driver, who would have to immediately take his vehicle to the garage after occurrence of the failure, more and more OEMs are now no longer accepting the "limp-aside" solution and demand a "fault tolerant" EPS system instead.

In order to enable such fault tolerant EPS systems, the sensor modules of the system have to provide not only ASIL D compliance, but they even have to sustain ASIL D rating in case of failure of one single failure. Thus, the sensor module has to provide redundant ASIL D compliance.

For fault-tolerance strategies, providing ASIL D compliant sensor ICs on system level will be a mandatory requirement for future OEM platforms in EPS applications

\section{Integrated Digital Linear Hall Sensor Concept with Dual Measurement Paths}

The main concept idea of this novel linear Hall sensor with a digital output protocol is to place at one single silicon die two redundant and diverse measurement path to sense the same magnetic physical quantity. Figure 2 is illustrating the top level diagram of this linear Hall sensor which is presented in this paper. All necessary blocks are implemented in redundant and in a diverse way. The power supply of the two signal paths use different supply voltage levels, the ADC is implemented differently and also the digital temperature and stress compensation is implemented in a diverse manner. All single common blocks (oscillator, digital part, EEPROM, Interface) contain several internal safety mechanisms to achieve the required ASIL rating. The clock-frequency can be checked via the interface protocol, the EEPROM contains error correction mechanism and the data is protected by CRCs additionally and the data transferred to the interface is protected by individual independent cyclic redundancy checks. To finally get enough independence also external safety mechanisms inside the connected ECU is used to finally complete the ASIL D compliance. This concept also provides the option to connect different single-wire interfaces like PSI5 [6], SENT [4] or SPC [5], which is an advantage when using a low pin-count leaded package for engine compartment applications.

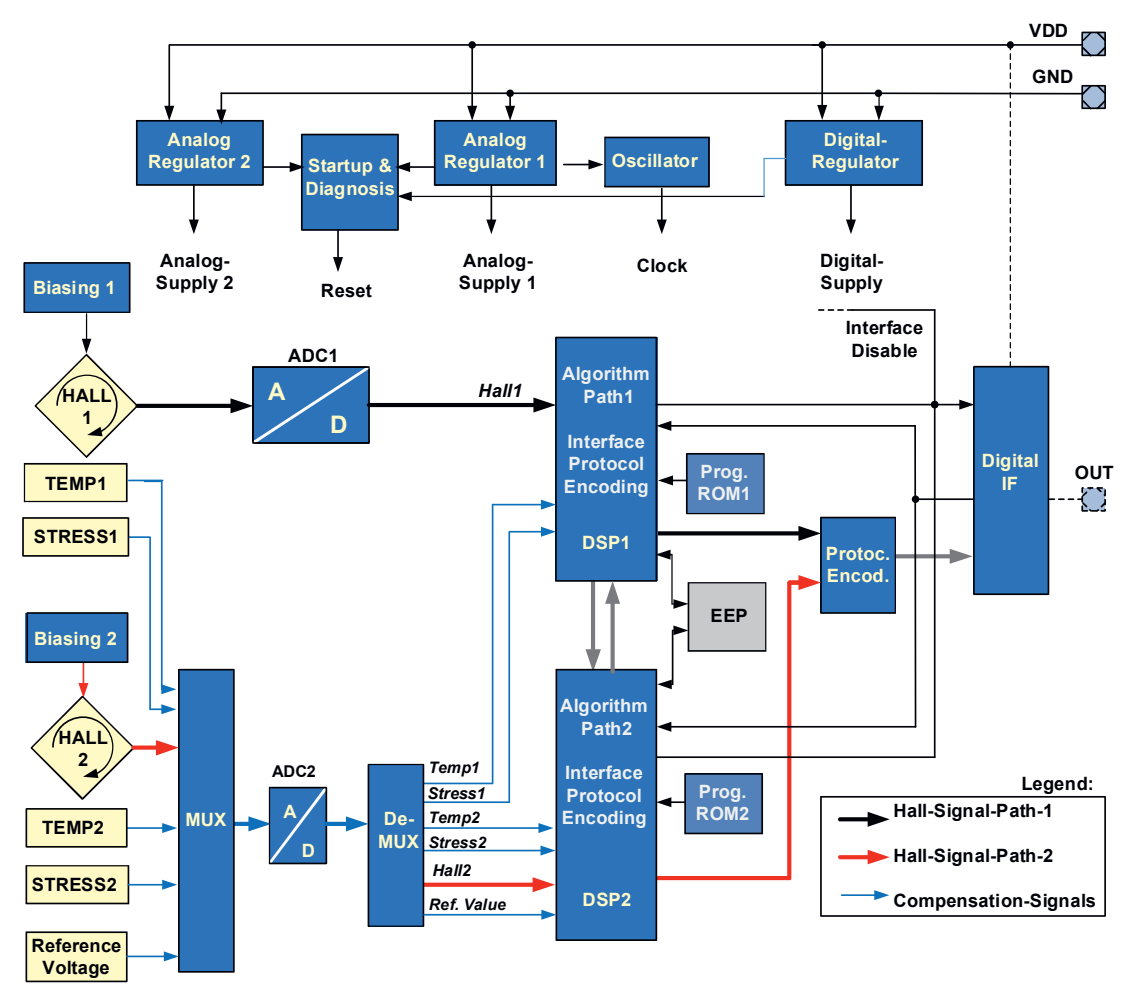

Fig. 2. Top-level block diagram of a monolithic digital linear Hall sensor with two measurement path and a digital output protocol [7] 


\section{Package, Pin-Out and Interface}

The linear Hall sensor has a digital output which can support different output protocols such as SPC [5], SENT [4] and PSI5 [6]. The linear Hall sensor fits into a very small package and needs only 3 pins. In case of PSI5 output the sensor data is transmitted by current modulation on the supply line, so only two wires are needed to connect the linear Hall sensor. In case of SENT or SPC interface type this sensor is operated in "open-drain" or "push-pull" using the 3 pins configuration with configurable protocol and timing parameters.

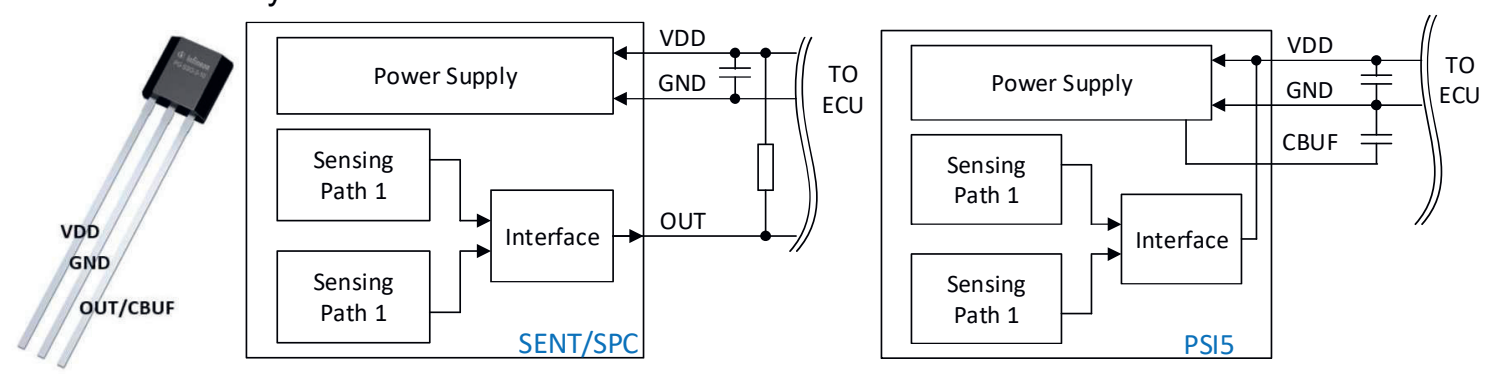

Fig. 3. Package drawing of ASIL D linear Hall sensor with a 3 pin digital output including connection possibilities

\section{Overview of Internal and External Safety Mechanism to achieve ASIL D Requirements}

In the following chapter the most important internal and external safety mechanism are described to achieve the ASIL D requirements on system level (SPFM $\geq 99 \%$ and $\mathrm{LFM} \geq 90 \%$ ) [1].

The safety analysis (FTA, DFA and FMEDA) showed, that several sensor failures have to be detected on silicon level internally to prevent white or gray areas on system level (for instance failure of the internal supplies, if undervoltage UV or overvoltage OV occur)

Tab. 1: Overview of the major internal safety mechanism [7]:

\begin{tabular}{|l|l|}
\hline $\begin{array}{l}\text { Name of internal } \\
\text { safety mechanism }\end{array}$ & \multicolumn{1}{|c|}{ Description } \\
\hline $\begin{array}{l}\text { Input ADC range } \\
\text { check }\end{array}$ & $\begin{array}{l}\text { Check of all ADC input ADC } \\
\text { signals (e.g. Hall-, } \\
\text { temperature and stress } \\
\text { values) }\end{array}$ \\
\hline $\begin{array}{l}\text { Internal } \\
\text { ROM/EEPROM } \\
\text { and RAM check }\end{array}$ & $\begin{array}{l}\text { Check of internal sensor } \\
\text { memory integrity via CRC } \\
\text { mechanism }\end{array}$ \\
\hline $\begin{array}{l}\text { Internal and } \\
\text { external supply } \\
\text { monitoring }\end{array}$ & $\begin{array}{l}\text { Check UV and OV } \\
\text { conditions regarding internal } \\
\text { and external supplies }\end{array}$ \\
\hline $\begin{array}{l}\text { Internal plausibility } \\
\text { checks between } \\
\text { path 1 and 2 }\end{array}$ & $\begin{array}{l}\text { Perform internal plausibility } \\
\text { checks regarding } \\
\text { temperature and stress } \\
\text { measurement between path } \\
1 \text { and 2 }\end{array}$ \\
\hline
\end{tabular}

The result of the safety analysis was to implement additional external safety mechanism on the ECU level to detect faults on system level. The following table shows the major external safety mechanism to reach des ASIL requirements on system level:

Tab. 2: Overview of the major external safety mechanism [7]:

\begin{tabular}{|l|l|}
\hline $\begin{array}{c}\text { Name of external safety } \\
\text { mechanism }\end{array}$ & \multicolumn{1}{c|}{ Description } \\
\hline EXT_SIGNAL_DIFF & $\begin{array}{l}\text { This mechanism } \\
\text { checks the } \\
\text { difference between } \\
\text { the two output } \\
\text { signal values }\end{array}$ \\
\hline EXT_PROTOCL_CHECKS & $\begin{array}{l}\text { Check external } \\
\text { digital output } \\
\text { protocol behavior } \\
\text { (e. g. CRC check } \\
\text { and timing of the } \\
\text { digital output } \\
\text { protocol) }\end{array}$ \\
\hline
\end{tabular}

The main external safety mechanism to achieve the ASIL D requirements on system level is the comparison between path 1 and 2 measurement output data of the sensor ASIC in the ECU. A key parameter for the diagnostic coverage is the remaining measurement uncertainty which was investigated during the development of this sensor system [8]. The following chapter shows in detail how the magnetic performance parameters (magnetic sensitivity drift, offset-drift and the output noise behavior over temperature and lifetime) are achieved.

\section{Overview of Verification Results}

The following figures show the achieved magnetic performance of this new linear Hall sensor with improved analog and digital 
compensation techniques of influencing parameters to the Hall sensitivity [9] [10]. Fig. 4 shows the achieved sensitivity drift and Fig. 5 the offset drift versus junction temperature of both implemented channels. Fig. 6 shows the achieved and intentionally different noise performance of both channels versus junction temperature because of diverse implementation.
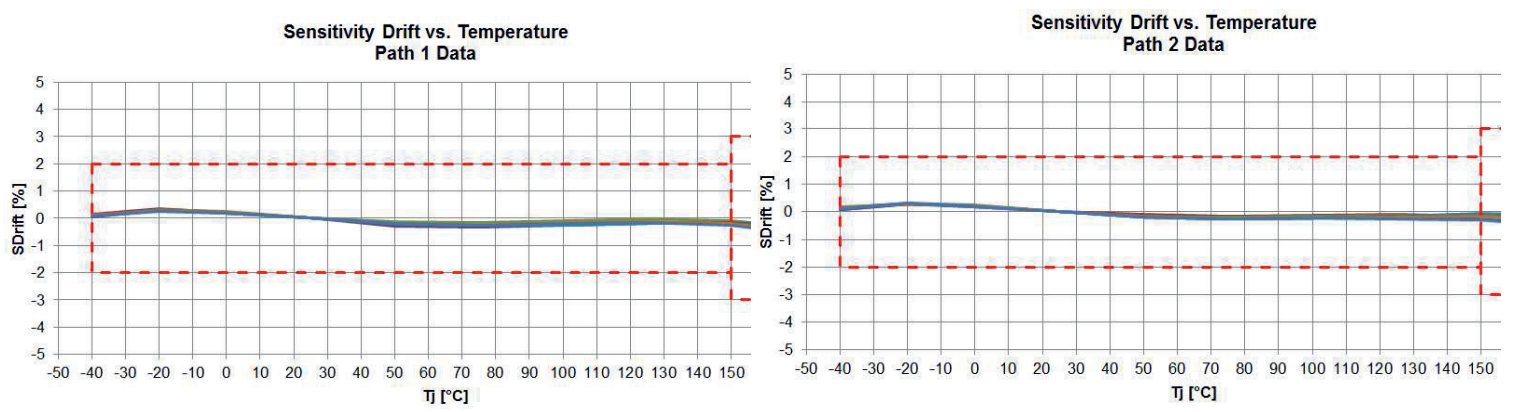

Fig. 4: $\quad$ Magnetic sensitivity drift of sensing path 1 and 2 versus junction temperature
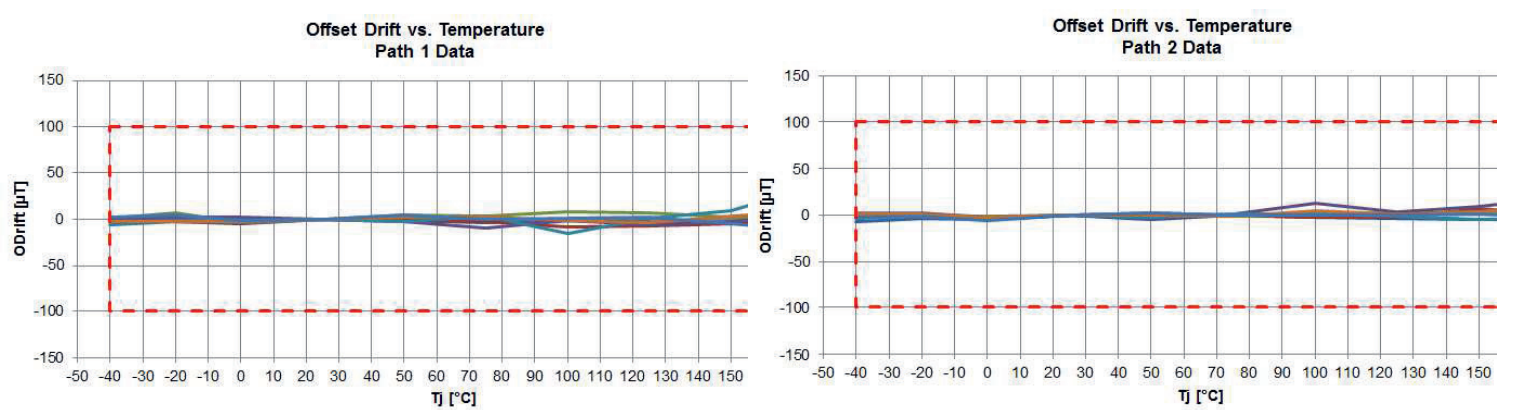

Fig. 5: Magnetic offset drift of sensing path 1 and 2 versus junction temperature
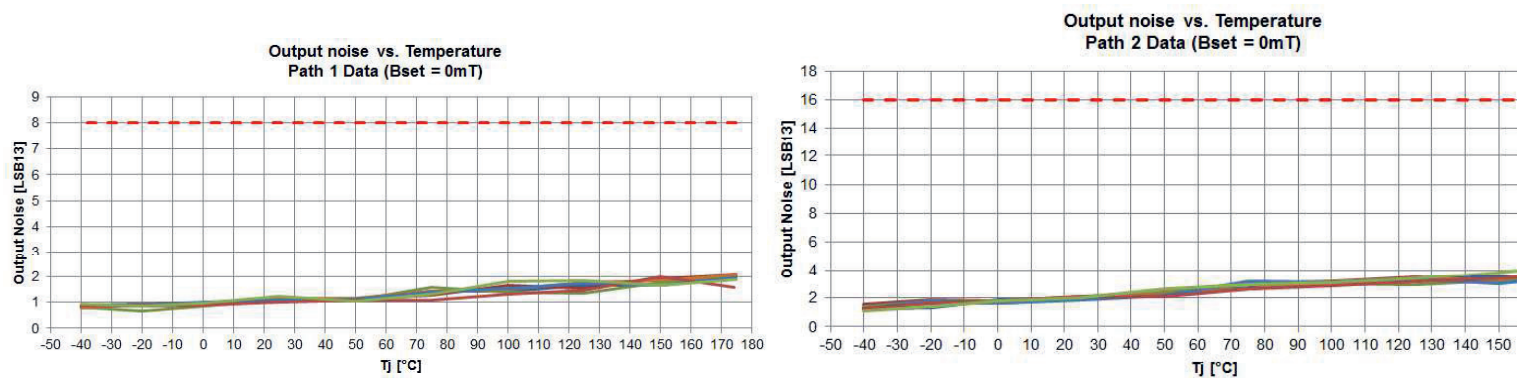

Fig. 6: $\quad$ Magnetic noise at $B=O m T$ of sensing path 1 and 2 versus junction temperature

\section{Summary}

In this paper an overview is shown how ASIL D according ISO 26262 requirements on system level (SPFM and LFM) with a dual measurement path approach on monolithic silicon die is achieved. For this purpose internal and external safety mechanism are introduced. During the safety analysis the main contributor was identified to reach these targets, the external signal comparison on system level in the ECU. For this main external safety mechanism, the magnetic performance of the presented Hall sensor was identified as key factors, to fulfill the overall safety requirements regarding ASIL D on system level.

\section{References}

[1] International Organization of Standardization, "ISO 26262 Road Vehicle Functional Safety", 15.11.2015

[2] Infineon Technologies AG, "TLE5012BD, GMRBased Dual Die Angle Sensor", Datasheet, Rev. $1.1,2015-03-12$ 
[3] Wolfgang Scheibenzuber, Franziska Moissl, Julao Nimler Elemparo, Ovaldo Borghetti, Harald Witschnig, Friedrich Rasbornig, "Dual-Sensor Package Technology Supporting ASIL-D in Safety-Related Automotive Applications", AMA Conference 19-21 May 2015, Nürnberg Germany, Proceedings SENSOR 2015, pp. 469 471

[4] SAE International J2716, "SENT - Single Edge Nibble Transmission for Automotive Applications", Rev. 4, 2016-04-29

[5] Infineon Technologies, "SPC - Short PWM Code", SPC2014 Specification Rev. 1.0, 2015-03-16

[6] PSI5 Standard Consortium, "Peripheral Sensor Interface", Technical Specification V2.1, 2012-1008

[7] Wolfgang Granig, Friedrich Rasbornig, Zettler Thomas, Michael Strasser, Mario Motz, Alessandro Michelutti „Redundant and Diverse Magnetic Field Digital Linear Hall Sensor

Definitions/Abbreviations:

$\begin{array}{ll}\text { ADC } & \text { Analog to Digital Converter } \\ \text { ASIL } & \text { Automotive Safety Integrity } \\ \quad \text { Level } & \\ \text { DFA } & \text { Dependent Failure Analysis } \\ \text { DSP } & \text { Digital Signal Processor } \\ \text { EEPROM } & \text { Electric Erasable }\end{array}$

\section{EPS}

Programmable Read Only Memory fails per hour)

FMEDA Failure Mode Effect and

Diagnostic Analysis
Concept for ASIL D Applications", SAE World Congress Experience, 4 - 6 April 2017, Detroit USA

[8] Wolfgang Granig, Dirk Hammerschmidt, Hubert Zangl "Calculation of Failure Detection Probability on Safety Mechanisms of Correlated Sensor Signals According to ISO 26262 ", SAE World Congress Experience, 4 - 6 April 2017, Detroit USA

[9] Udo Ausserlechner, Mario Motz, Michael Holliber, "Compensation of the Piezo-Hall Effect in Integrated Hall Sensors on (100)-Si" IEEE Sensors Journal, Vol. 7, No. 11, 1475-1482, November 2007.

[10] Mario Motz, Udo Ausserlechner "Electrical Compensation of Mechanical Stress Drift in Precision Analog Circuits", Book: Wideband Continuous Time SD ADCs, Automotive Electronics, and Power Management, Springer International Publishing Switzerland 2016, Chapter 16

$\begin{array}{ll}\text { FTA } & \text { Fault Tree Analysis } \\ \text { LFM } & \text { Latent Fault Metric } \\ \text { OV } & \text { Over voltage } \\ \text { PSI5 } & \text { Peripheral Sensor Interface for }\end{array}$
Automotive Applications

$\begin{array}{ll}\text { ROM } & \text { Read Only Memory } \\ \text { SENT } & \text { Single Edge Nibble }\end{array}$ Transmission

SPC Short PWM Code, Infineon enhancement to SENT

SPFM Single Point Fault Metric

UV Under voltage 